\title{
A case for the smart use of smartwatch-based technologies
}

\author{
Tommaso Sanna \\ Fondazione Policlinico A. Gemelli IRCCS, Università Cattolica del Sacro Cuore, Institute of Cardiology, Rome, Italy \\ Correspondence to: Tommaso Sanna, MD. Fondazione Policlinico A. Gemelli IRCCS, Università Cattolica del Sacro Cuore, Institute of Cardiology, \\ Largo A. Gemelli 8, 00168 Rome, Italy. Email: tommaso.sanna@unicatt.it. \\ Provenance: This is an invited Editorial commissioned by the Section Editor Fang-Zhou Liu (Guangdong Cardiovascular Institute, Guangzhou, \\ China). \\ Comment on: Bumgarner JM, Lambert CT, Hussein AA, et al. Smartwatch Algorithm for Automated Detection of Atrial Fibrillation. J Am Coll \\ Cardiol 2018;71:2381-8.
}

Submitted Aug 02, 2018. Accepted for publication Aug 28, 2018.

doi: $10.21037 /$ jtd.2018.08.141

View this article at: http://dx.doi.org/10.21037/jtd.2018.08.141

The "Internet of Things" is connecting doctors with patients in previously unimaginable ways. In their recent manuscript, Bumgarner et al. investigated a possible role of a smartwatch-based technology (SBT) (Apple Smartwatch by Apple, Cupertino, California, USA bundled with the Kardia Band by AliveCor, Mountain View, California, USA) in the management of patients scheduled for cardioversion (1). Briefly, this SBT was used to acquire a rhythm strip and automatically assess whether atrial fibrillation (AF) was present before and after elective cardioversions (CV). In more detail, in this blinded and perspective study, patients were trained to self-record single-lead rhythm strips of $30 \mathrm{sec}$. duration before and after elective $\mathrm{CV}$ using the AliveCor Kardia wristband paired to an Apple Smartwatch via Bluetooth technology; these SBT-acquired recordings were processed in real-time by a dedicated App which, through a proprietary algorithm, assessed whether AF was present. At the same time, i.e., pre- and post-cardioversion, standard 12-lead electrocardiogram (EKG) were also recorded, interpreted by a cardiologist and used as the gold standard for the diagnosis of AF. In this study, the SBT was able to automatically interpret and classify around twothirds of the SBT recordings while one-third remained unclassified. Among the automatically-interpretable recordings, the SBT classified AF with $93 \%$ sensitivity and $84 \%$ specificity. Among the non-automatically-interpretable SBT recordings, a physician was still able to diagnose AF on SBT recordings with $100 \%$ sensitivity and $80 \%$ specificity. The authors agreeably concluded that SBT, supported by physician review of the recordings, can reliably differentiate AF from sinus rhythm avoiding scheduling un-necessary electrical CVs. This is a clinically useful finding. However, there is more in this study than meets the eye. In fact, the most remarkable result of the study is that it adds further evidence in favour of using smartwatchbased technologies in the management of the worldwide epidemic of AF. Fantasy is the only limit to the possible applications of SBT to detect AF: first detection of AF in the setting of primary prevention programs to prevent $\mathrm{AF}$ complications such as heart failure and ischemic stroke (2); first detection of $\mathrm{AF}$ in secondary prevention programs after thromboembolic events such as in cryptogenic stroke and embolic stroke of unknown source (ESUS); modulation of drug treatment in patients with known AF; detection of recurrences after AF ablation; end-point evaluation in clinical trials; and many others. Thus, SBT add with full rights to the other tools so far tested to pursue the diagnosis of $\mathrm{AF}$, including short term external monitoring devices, external long-term monitoring devices and cardiac implantable electronic devices (CIEDs), i.e., pacemakers, implantable cardiac defibrillators and insertable cardiac monitors. Yet, the study from Bumgarner et al. shows that technical limitations to SBT-based management of AF do exist. The frequent occurrence of non-automatically interpretable SBT recordings and suboptimal sensitivity and specificity are the most significant limitations of the technology and should be properly addressed. In fact, tracing review is a resource-consuming process, especially in primary prevention programs where large populations are involved. However, technical limitations will eventually 

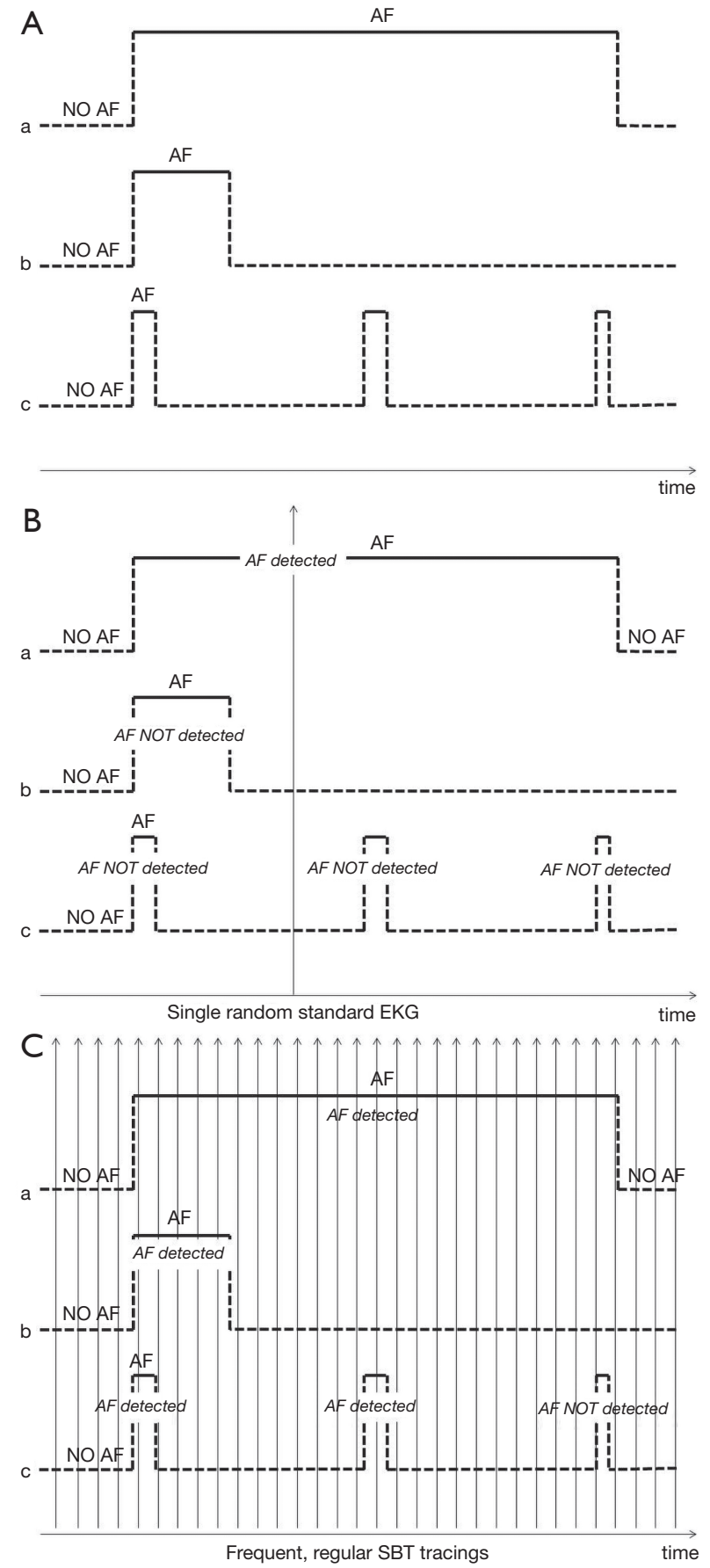

Figure 1 Atrial fibrillation (AF) pattern and AF detection by different electrocardiographic techniques. (A) Different patterns of AF are shown. Pattern a: long AF episode; Pattern b: short AF episode; Pattern c: multiple very short AF episodes. All patterns are detected by longterm continuous monitoring techniques; (B) AF detection with single standard electrocardiogram (EKG). Pattern a: detected; Pattern b: not detected; Pattern c: not detected; (C) AF detection with multiple regular smartwatch based technology (SBT) recordings: Pattern a: detected; Pattern b: detected; Pattern c: some episodes detected, some not. be overcome with research and development. The true challenge of SBT detection of AF is not technical but clinical. In fact, when assessing the role of novel monitoring devices to pursue the diagnosis of AF, we should always bear in mind that evidence supporting the benefit of anticoagulation in patients with non-valvular AF originates from studies in which AF was diagnosed with standard electrocardiography. External long-term monitoring devices or CIEDs, with their uninterrupted monitoring, detect every single episode of AF irrespective of duration (Figure $1 A$ ), and actually most episodes of AF or of atrial high rate episodes (AHREs) detected are short-lasting (3). On the other hand, standard electrocardiography, due to the limited time window of the recording and the low frequency of sampling (e.g., one recording of 30 seconds per year), detects with a higher probability asymptomatic episodes of $\mathrm{AF}$ of longer duration, i.e., long episodes of paroxysmal AF or persistent AF (Figure 1B). Therefore, the applicability of findings supporting the benefit of anticoagulation in patients in whom the diagnosis was obtained by standard electrocardiography (biased in favour of long AF episodes) to patients in whom the diagnosis is made by long-term continuous monitoring devices (with many short-lasting episodes) is uncertain. In order to fill this gap of knowledge, several randomized clinical trials are currently assessing whether oral anticoagulants offer any benefit over aspirin or placebo in patients with less than 24 hours of $\mathrm{AF}$ or AHREs $(4,5)$. However, the unique relation between AF burden and AF detection with SBT, characterized by a high frequency of sampling and recordings of short duration (e.g., one 30 sec. recording per day per 365 days/year), is difficult to unravel (Figure 1C). A randomized trial using $\mathrm{SBT}$ to diagnose $\mathrm{AF}$ in a primary prevention setting on over 1,000 patients showed that SBT was superior to routine care in detecting $\mathrm{AF}$, but was not powered to demonstrate a reduction in thromboembolic events despite anticoagulation in patients with AF detected (2). SBT detection of AF will soon be technically ready for prime time. The medical community is about to be overwhelmed by an epidemic of SBT-detected AF. However, whether patients with SBTdetected AF in primary or secondary prevention settings benefit from anticoagulation, though conceivable, is currently undemonstrated and this gap of knowledge should be rapidly addressed by properly designed clinical studies.

\section{Acknowledgements}

None. 


\section{Footnote}

Conflicts of Interest: The author is consultant to Medtronic and received speaker fees from Medtronic, BoehringerIngelheim, BMS-Pfeizer and Abiomed.

\section{References}

1. Bumgarner JM, Lambert CT, Hussein AA, et al. Smartwatch Algorithm for Automated Detection of Atrial Fibrillation. J Am Coll Cardiol 2018;71:2381-8.

2. Halcox JPJ, Wareham K, Cardew A, et al. Assessment of Remote Heart Rhythm Sampling Using the AliveCor Heart Monitor to Screen for Atrial Fibrillation: The REHEARSE-AF Study. Circulation 2017;136:1784-94.

Cite this article as: Sanna T. A case for the smart use of smartwatch-based technologies. J Thorac Dis 2018;10(Suppl 33):S3875-S3877. doi: 10.21037/jtd.2018.08.141
3. Sanna T, Diener HC, Passman RS, et al. Cryptogenic stroke and underlying atrial fibrillation. $\mathrm{N}$ Engl J Med 2014;370:2478-86.

4. Lopes RD, Alings M, Connolly SJ, et al. Rationale and design of the Apixaban for the Reduction of ThromboEmbolism in Patients With Device-Detected SubClinical Atrial Fibrillation (ARTESiA) trial. Am Heart J 2017;189:137-45.

5. Kirchhof P, Blank BF, Calvert $M$, et al. Probing oral anticoagulation in patients with atrial high rate episodes: Rationale and design of the Non-vitamin K antagonist Oral anticoagulants in patients with Atrial High rate episodes (NOAH-AFNET 6) trial. Am Heart J 2017;190:12-8. 